\title{
DESIGNING OF ZONE TARIFF IN INTEGRATED TRANSPORT SYSTEMS
}

When designing a tariff system, there are several possibilities how to design the tariff. One of them is dividing the region into the zones. These zones are usually created by stops in one or more municipalities and the price of travelling between arrival and destination stops is determined by the number of travelled tariff zones by the passenger. There are various approaches how to design prices in such a system. In this article we introduce a mathematical model of the tariff zones design based on counting zones and introducing two approaches for designing prices within this system. We will focus on solving this problem using a universal optimisation tool Xpress, on data set of the selected region. We will compare both approaches in terms of solution quality and computational time.

Keywords: Tariff planning, tariff zones design, IP solver, location problem, p-median problem.

\section{Introduction}

An integrated transport system is the way how to provide transport service in the region by integration of all modes of transport. The goal of integrated transport system in the region is to increase the number of transported passengers, improve the proportion between individual and public transport in favour of public transport, better coordination between all modes of transport and creating the tariff system which enables to use one ticket for all modes of transportation in the region [1].

The design of such systems is connected with the solving of many optimisation tasks such as coordination of connections in transport nodes, optimisation of connection supply, minimisation of time losses related to the changing of travel connection and the design of the tariff configuration.

When designing a tariff system, it is important to create it so that it should be sufficiently attractive for passengers and efficient for carriers. There are several ways of creating a tariff system. One of them is dividing the region into the tariff zones [2].

The paper is organized as follows. In the chapter 2 and 3 we analyse the zone tariff system design problem and the possibilities of setting fair prices for travelling. In the fourth chapter we introduce a mathematical model of the tariff zones design based on counting zones and two ways how to design the prices in such a system.

In the chapter 5 we will focus on the solution of this problem using a universal optimisation tool XPRESS. We will compare approaches for price design in terms of solution quality.

\section{Designing the tariff system in integrated transport systems}

There are several possibilities how to design tariff in public transportation. As mentioned in [2], the basic and frequently used way is a distance tariff system where the price for a trip depends on the length of the trip. This tariff system is mostly considered as fair. If we want to calculate the price for the trip, we need to know the distance between the starting and destination stations of the trip.

Another possibility is the unit tariff. It is the simplest tariff system. In this case all trips cost the same price and are independent on their length. The unit tariff is frequently used in city public transport, but it is not very suitable for regional public transportation, especially for large regions. Between the unit tariff and the distance tariff there is a zone tariff system. In this system the whole area has to be divided into smaller sub-regions - the tariff zones. If the price is given arbitrarily for each pair of zones, the tariff system is called a zone tariff with arbitrary prices. An example of this tariff system is, for instance, the Zilina Regional Integrated Transport System in the Slovak Republic or IREDO system in Pardubice and Hradec Kralove region in the Czech Republic, see Fig. 1.

Another possibility of the zone tariff system is the counting zone tariff system. The price of a trip in this system is calculated according to the number of travelled zones in the trip. The prices in this system are dependent on the starting and destination zones of the trip, but the trips passing the same number of zones are the same price. An example of the counting zone tariff system in Zurich region in Switzerland is in Fig. 2.

\footnotetext{
* Michal Kohani

Department of Transportation Networks, Faculty of Management and Informatics, University of Zilina, Slovakia,

E-mail: michal.kohani@fri.uniza.sk
} 


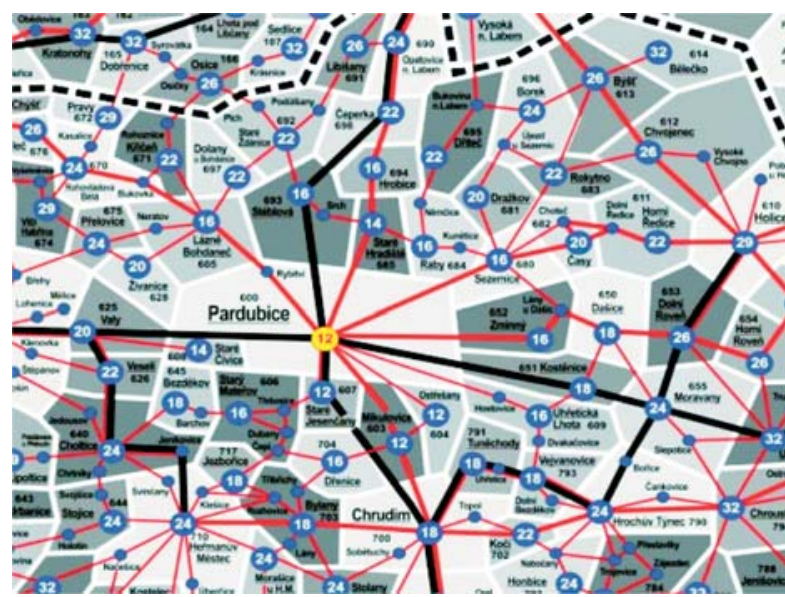

Fig. 1 Prices in IREDO system (www.iredo.cz)



Fig. 2 Zone tariff in integrated transportation system in Zurich region, Switzerland (www.zvv.ch)

\section{Designing prices in the zone tariff system}

A number of objectives must be respected when changing the existing tariff system to zone tariff system. To avoid a significant increase in prices for passengers and also a decrease in revenues for carriers, the goal often is to design the zones in the way that the new and the old prices for most of the trips are as close as possible. This means that neither the public transportation company nor the customers will have major disadvantages when changing the current tariff system to a zone tariff. Another goal can be to design fair zones. In this case we do not consider the deviation to some old prices, but the deviation from a reference price, for instance one which is considered to be fair, like the distance tariff. In this approach, the public transportation company needs to estimate its new income [2] [3]. There are more possibilities, how to set a new price for travelling. In this article, we will focus only on two of them.

In the first case (Price_uni), the unit price $f$ for travelling in one zone will be established. This means that for the calculation of the new prices it will be necessary to count the number of travelled zones and multiplied it by the unit price for one zone.

In the second case (Price_dif) two different unit prices will be determined - price $f_{1}$ per travelling in the first zone and unit price $f_{2}$ for travelling in each additional zone. The final price will be calculated as a sum of the basic price for the first zone and number of other travelled zones multiplied by the unit price for additional zones.

\section{Model of the tariff zones design problem}

In the available literature we found mostly the heuristic approaches to solve of the tariff zones design without any exact formulation [2]. We will formulate the mathematical model of the problem according to the model mentioned in [3]. When creating the mathematical model of the problem, we were inspired by the model of $p$-median problems.

Let all the stations in the network of public transport constitute the set $I$. The stations $i$ and $j$ from set $I$ are connected by the edge $(i, j) \in V$, if there is a direct connection by a public transport line between these two stations. Symbol $V$ denotes the set of edges. The distance between stations $i$ and $j$ is denoted as $d_{i j}$. For each pair of stations $i$ and $j$ is $c_{i j}$ the current price of travelling between these two stops. The number of passengers between stations $i$ and $j$ is $b_{i j}$ (OD matrix).

As mentioned above, if we want to calculate the new price of the trip between nodes $i$ and $j$ in the counting zones tariff system, we need to calculate how many zones are crossed on this trip. The calculation of the number of crossed zones can be easily replaced by the calculation of crossed zone borders. We assume that the node can be assigned only to one zone and then the border between zones is on the edge. We will introduce the binary variable $w_{r s}$ for each existing edge $(r, s) \in V$, which is equal to 1 if stations $r$ and $s$ are in different zones and is equal to 0 otherwise.

For calculation of the number of crossed borders we need to determine the used path for travelling between stations $i$ and $j$. We introduce $a_{i j}^{r s}$, where the used paths will be observed. $a_{i j}^{r s}$ is equal to 1 if the edge $(r, s)$ will be used for travelling between $i$ and $j$ and 0 otherwise. This calculation will be done before solving the model.

The current or fair price between stations $i$ and $j$ is denoted by $c_{i j}$. The new price determined by Price_uni definition for given value of parameter $f$ will be calculated as follows (1): 


$$
n_{i j}=f \cdot\left(1+\sum_{(r, s) \in V} a_{i j}^{r s} w_{r s}\right)
$$

The new price determined by Price_dif definition for given values of parameters $f_{1}$ and $f_{2}$ will be calculated as follows (2):

$$
n_{i j}=f_{1}+\sum_{(r, s) \in V} f_{2} a_{i j}^{r s} w_{r s}
$$

We assume that the node can be assigned exactly to one zone Then we can introduce binary variables $y_{i}$, which represent the "fictional" centre of the zone. Variable $y_{i}$ is equal to 1 if there is a centre of the zone in node $i$ and 0 otherwise. For each pair of stations $i$ and $j$ we introduce variables $z_{i j}$. Variable $z_{i j}$ is equal to 1 if the station $j$ is assigned to the zone with centre in the node $i$ and 0 otherwise. We expect to create at most $p$ tariff zones.

According to [2] and [3] we introduce two different objective functions. The first one will be the maximal deviation between the current or fair price and new price determined by the number of crossed zones for all passengers between $i$ and $j$. The second one will be the average deviation between the current and new price for all passengers.

The mathematical model for criterion maximal deviation can be written in the form (Model Dev_max):

$$
\begin{aligned}
& \text { Minimise } d e v_{\max }=\max \left\{b_{i j}\left|c_{i j}-n_{i j}\right|, i, j \in I\right\} \\
& \text { subject to } \sum_{i \in I} z_{i j}=1 \text { for } j \in I \\
& z_{i j} \leq y_{i} \text {, for } i, j \in I \\
& z_{i j}-z_{i k} \leq w_{j k} \text {, for } i \in I,(j, k) \in V \\
& \sum_{i \in I} y_{i} \leq p \\
& z_{i j} \in\{0,1\} \text {, for } i, j \in I \\
& y_{i} \in\{0,1\}, \text { for } i \in I \\
& w_{i j} \in\{0,1\}, \text { for }(i, j) \in V
\end{aligned}
$$

The mathematical model for criterion of average deviation can be written in the form (Model Dev_avg):

$$
\text { minimise } d e v_{\text {avg }}=\frac{\sum_{i \in I} \sum_{j \in J}\left|c_{i j}-n_{i j}\right| b_{i j}}{\sum_{i \in I} \sum_{j \in J} b_{i j}}
$$

Conditions (4) ensure that each station will be assigned exactly to only one zone. Conditions (5) ensure that the station $j$ will be assigned only to the existing centre of the zone. Conditions (6) are coupling conditions between variables for allocation of the station to the zone and the variables for determining the zone border on the edge $(j, k)$. If stations $j$ and $k$ are in different zones then the value of variable $w_{j k}$ must be equal to 1 . Condition (7) ensures that we will create maximally $p$ zones.

Both models can be easily solved using universal IP solver, so we will obtain the exact solution of the problem. As in the model with criterion maximal deviation the objective function (3) is not a linear function, we need to modify this objective function to linear form. Reformulation of this model is mentioned in [3].

To determine the optimal values of parameters in the model, we used a two-phase procedure. In the first phase we determine the optimal number of zones. In the second phase, for the given number of zones $p$, we repeatedly solve models with different settings of parameters $f, f_{1}$ and $f_{2}$ respectively. As the optimal we choose the solution of the model and parameters setting with the smallest value of the objective function.

\section{Numerical experiments}

Verification of both models presented above was made on the data of the Zvolen County in the Slovak Republic. The stations in the network are represented by the 51 municipalities or parts of municipalities. The simplified scheme of this public transport network is in Fig. 3.

In each node the main station was selected to calculate the distance matrix and the OD matrix. Current prices are distance prices and were calculated according to real prices for travelling by regional buses in this area.

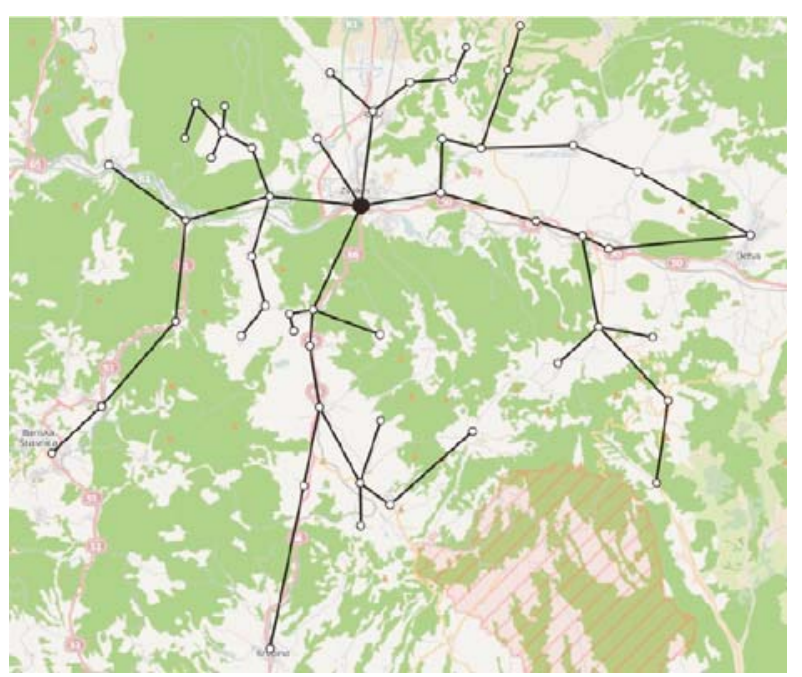

Fig. 3 Public transport network in Zvolen County (Map base: openstreetmap.org)

The OD matrix was estimated using the quadratic gravity model [4] where the number of passengers between nodes $i$ and $j$ is calculated as follows: 
$\frac{b_{i} b_{j}}{d_{i j}}$

Parameter bi represents the number of inhabitants served by the node $i$. These data were obtained from the database of the population in the Slovak Republic in 2008, which is available at our department. Parameter $p$ was set to 4 . The goal of experiments was to compare quality of solutions achieved using two variants of the model, and two new variants of setting the prices. We also wanted to compare the computation time.

To perform the experiments we used the universal optimisation software tool XPRESS 7.3 [5] [6]. The experiments were performed on a personal computer equipped with Intel Core 2 Duo E6850 with parameters $3 \mathrm{GHz}$ and $3.5 \mathrm{~GB}$ RAM.

For all the experiments with Price_dif we set the values of parameter $f_{1}$ from 0.1 to 1 with step by 0.1 and values of parameter $f_{2}$ were from 0.1 to 1.5 with step by 0.1 . Together 150 instances of each model were solved. For all the experiments with Price_uni we set the values of parameter $f$ from 0.1 to 1.5 with step by 0.1 . In this case 15 instances of each model were solved.

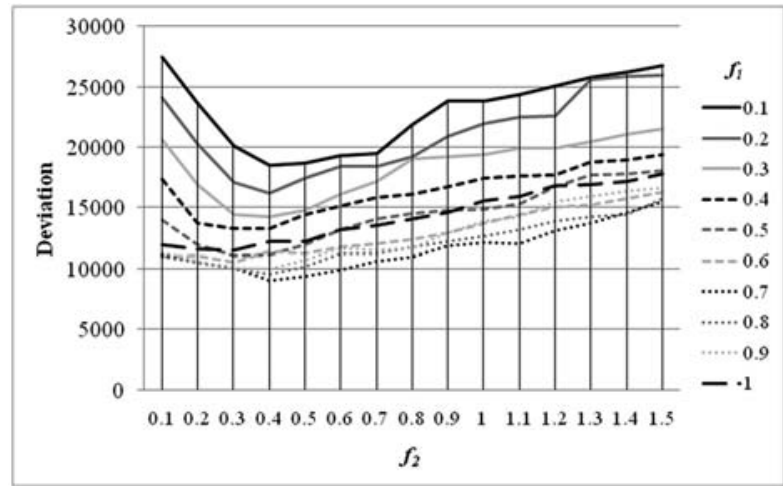

Fig. 4 Results for Price_dif using Dev_max model for different setting of parameter $f_{l}$

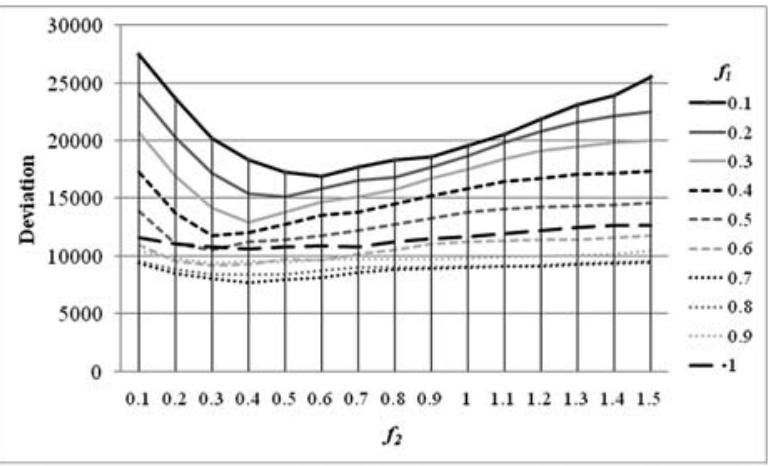

Fig. 5 Results for Price_dif using Dev_avg model for different setting of parameter $f_{1}$
In Fig. 4 are results for Price_dif using Dev_avg model. Each line in the graph represents one setting of parameter $f_{1}$. In Fig. 5 are results for Price_dif using Dev_max model. Each line in the graph represents one setting of parameter $f_{1}$. In Figs. $4-6$ are calculated total deviations between the current prices and new prices for all passengers to compare the quality of solution.

From the results we achieved for the price setting Price_dif, we select the best results for parameter $f_{1}$ achieved using Dev_avg model $\left(f_{1}=0.7\right)$ and Dev_max model $\left(f_{1}=0.7\right)$. These best results were compared with the results we achieved by using the Price_uni setting of prices solved by both models. Results are shown in Fig. 6.

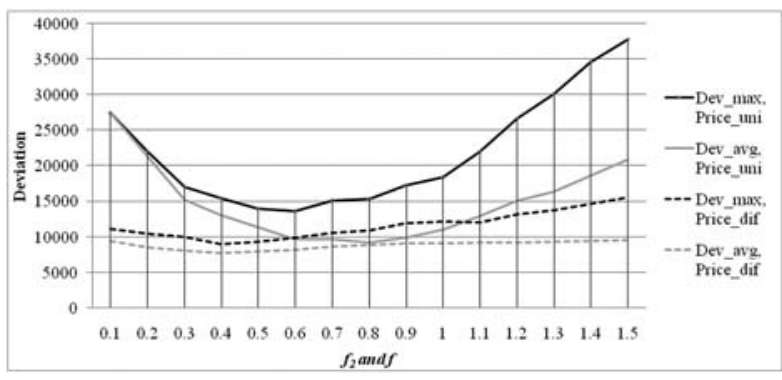

Fig. 6 Comparison of the results for Price_uni with different values of parameter $f$ and the best results for parameter $f_{l}\left(f_{1}=0.7\right)$ for Price_dif

Average computational time of Dev_avg model was 14.57 seconds and the maximal computational time was 122.68 seconds. The average computational time in the case of Dev_max model was 3.45 seconds and the maximal computational time was 12.65 seconds.

\section{Conclusions}

The results of numerical experiments show that the computational time is acceptable. We can obtain good proposal of tariff zones in the integrated transport system when solving this model.

When comparing the quality of solutions for both models with Price_dif price setting we obtain the best results for the value of parameter $f_{1}$ equal to 0.7 and the model Dev_avg, as we can see in Fig. 4 and Fig. 5. When we compare best results obtained with Price_dif price setting with results obtained with Price_uni price setting for both models, we can see that Price_dif price setting with Dev_avg model gives us the best solution for given value of $p$. Also in the literature [2] is the use of the Dev_avg model accepted by experts in the field.

In terms of the best result itself the value 7691 of total deviation is acceptable for the size of the region. In real conditions it would be necessary to decide about the final proposal of zones by the participating operators and local authorities. 
Because of the low computational time this approach allows us studying various settings and comparison of model parameters which can be advantageous in practical usage of this model. In the future we want to study also other different objective functions and compare this approach with other methods, for example heuristic methods mentioned in [7].

Acknowledgment: This work was supported by the research grants VEGA 1/0296/12 "Public Service Systems with Fair Access to Service" and APVV-0760-11 "Designing of Fair Service Systems on Transportation Networks".

We would also like to thank to "Centre of excellence for systems and services of intelligent transport" (ITMS 26220120028) for building up the infrastructure, which was used.

\section{References}

[1] HAJHALOVA, B.: Kedy sa stanu integrovane dopravne systemy osobnej dopravy na Slovensku realitou? [When the integrated transportation systems in passenger transport become a reality in the Slovak Republic?], Doprava a spoje : Internetovy casopis 1, 2011, pp. 29-36

[2] SCHOBEL, A.: Optimization in Public Transportation: Stop Location, Delay Management and Tariff Zone Design in a Public Transportation Network. Springer, 2006

[3] KOHANI, M.: Exact Approach to the Tariff Zones Design Problem in Public, Proc. of the $30^{\text {th }}$ intern. conference: Mathematical methods in economics, Karvina, September 2012, Silesian University in Opava, School of Business Administration, 2012, pp. 426-431.

[4] CENEK, P., JANACEK, J., JANOSIKOVA, L.: Location of Transportation Districts at Modeling of Tranportation Processes in a Region. Communications - Scientific Letters of the University of Zilina 4, No. 1-2, 2002, pp. 5-9.

[5] Mosel Language Reference Manual, Fair Isaac Corporation, 2010, Birmingham, p. 22.

[6] XPRESS-Optimizer Reference Manual. Fair Isaac Corporation, 2010, Birmingham, p. 549.

[7] BABEL. L., KELLERER, H.: Design of Tariff Zones in Public Transportation Networks: Theoretical Results and Heuristics, Mathematical Methods of Operations Research 58 2003, Springer-Verlag, pp. 359-374. 